\title{
CORRECTION
}

\section{Correction to: Long-Term Effect of Immediate Versus Delayed Fingolimod Treatment in Young Adult Patients with Relapsing-Remitting Multiple Sclerosis: Pooled Analysis from the FREEDOMS/FREEDOMSII Trials}

Angelo Ghezzi · Tanuja Chitnis · Annik K-Laflamme ·

Rolf Meinert · Dieter A. Häring · Daniela Pohl

Published online: February 14, 2020

(c) The Author(s) 2020

Correction to: Neurol Ther (2019) 8:461-475

https://doi.org/10.1007/s40120-019-0146-z

Figures $1 \mathrm{a}, 1 \mathrm{~b}$ and 3 were published incorrectly in the original version. The corrected images are given as follows.

The original article can be found online at https://doi. org/10.1007/s40120-019-0146-z.

\section{A. Ghezzi $(\bowtie)$}

Centro Studi Sclerosi Multipla, Gallarate, Italy

e-mail: angelo.ghezzi@asst-valleolona.it

T. Chitnis

Partners Pediatric Multiple Sclerosis Centre,

Massachusetts General Hospital, Boston, MA, USA

A. K-Laflamme · D. A. Häring

Novartis Pharma AG, Basel, Switzerland

R. Meinert

DATAMAP GmbH, Freiburg, Germany

D. Pohl

Division of Neurology, Children's Hospital of

Eastern Ontario, Ottawa, ON, Canada 
(a) $6 \mathrm{~m}-\mathrm{CDI}$

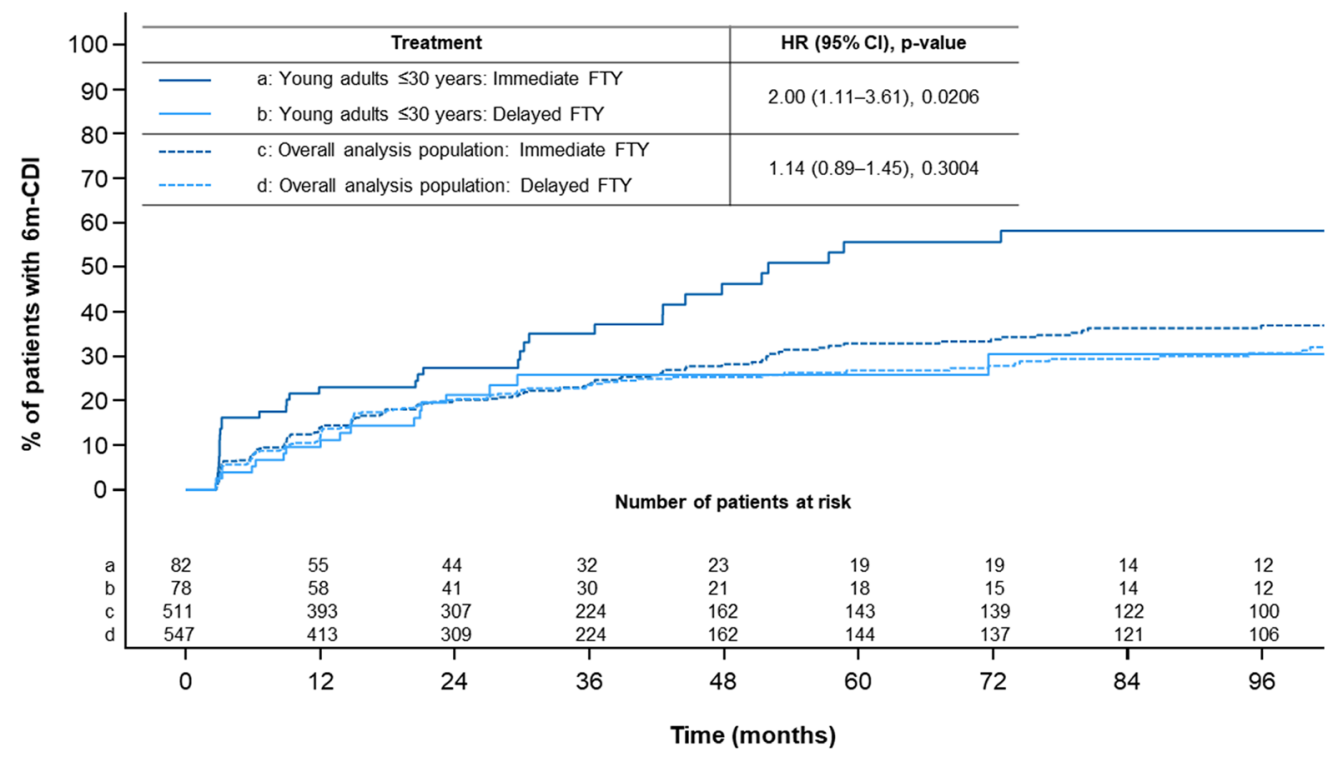

(b) $6 \mathrm{~m}-\mathrm{CDI}+$

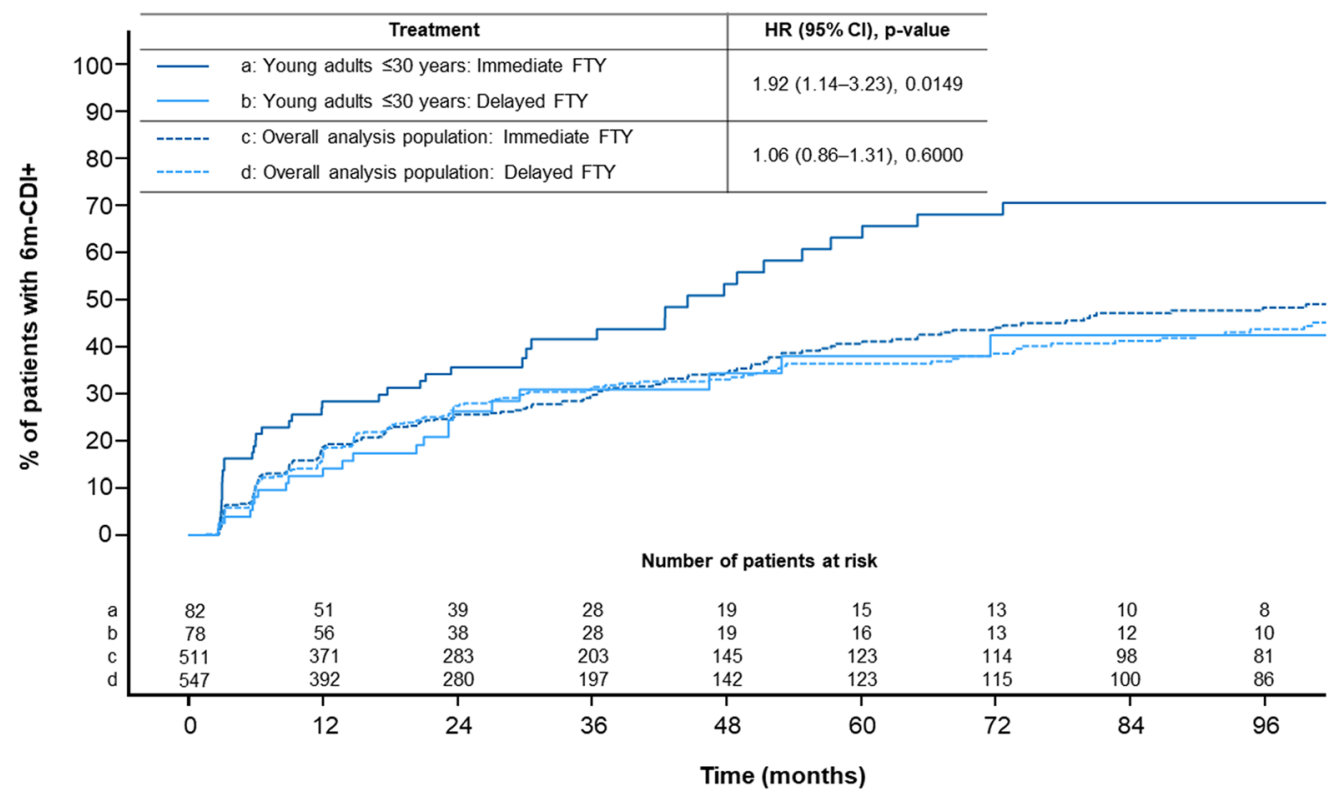

Fig. 1 Kaplan-Meier estimates of time to $6 \mathrm{~m}$-CDI (a) and $6 \mathrm{~m}-\mathrm{CDI}+(\mathbf{b})$ in young adult patients and the overall analysis population from pooled FREEDOMS/ FREEDOMS II studies. The Cox regression model was adjusted for sex, age, baseline EDSS and number of relapses in the 2 years prior to the study. Subjects with baseline
EDSS score $<2.0$ were excluded for $6 \mathrm{~m}$-CDI. Subjects with baseline EDSS score $\leq 2.0$ were excluded for $6 \mathrm{~m}$ $\mathrm{CDI}+6 \mathrm{~m}$-CDI 6-month confirmed disability improvement, 6m-CDI+ 6-month confirmed disability improvement-plus, CI confidence interval, EDSS Expanded Disability Status Scale, FTY fingolimod, HR hazard ratio 


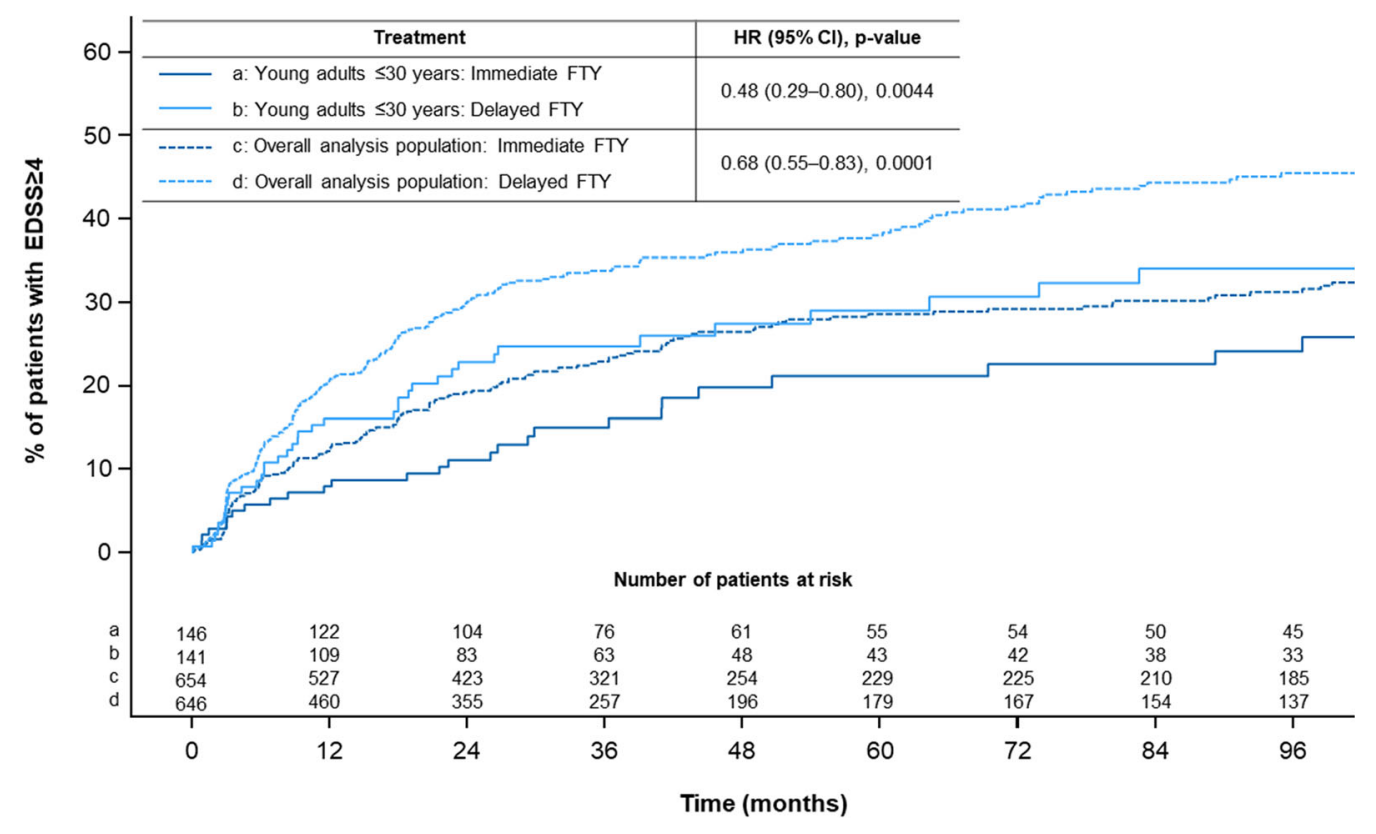

Fig. 3 Time to EDSS $\geq 4.0$ in young adult patients and the overall analysis population from pooled FREEDOMS/ FREEDOMS II studies. The Cox regression model was adjusted for sex, age, baseline EDSS and number of relapses

Open Access. This article is distributed under the terms of the Creative Commons Attribution-NonCommercial 4.0 International License (http://creativecommons.org/licenses/ by-nc/4.0/), which permits any noncommercial use, distribution, and reproduction in any in the 2 years prior to the study. Subjects with baseline EDSS score $\geq 4.0$ were excluded. $C I$ confidence interval, EDSS Expanded Disability Status Scale, FTY fingolimod, $H R$ hazard ratio medium, provided you give appropriate credit to the original author(s) and the source, provide a link to the Creative Commons license, and indicate if changes were made. 